\title{
"In x Out": Revisando o Viés de Grupo através da Perspectiva Biológica
}

\author{
Victor Kenji Medeiros Shiramizu ${ }^{1}$ \\ Maria Emília Yamamoto \\ Programa de Pós-Graduação em Psicobiologia da Universidade Federal \\ do Rio Grande do Norte, Natal, RN, Brasil
}

\begin{abstract}
Resumo
Os fatores subjacentes ao favorecimento do endogrupo e de indiferença/hostilidade ao exogrupo são amplamente estudados pela psicologia social, onde estudos reportam que, por exemplo, indivíduos confiam mais e associam palavras positivas aos membros do mesmo grupo. $\mathrm{O}$ estudo desses fatores pode ajudar na compreensão de fenômenos tais como preconceito e etnocentrismo. As perspectivas evolucionista e neuroendócrina vêm sendo extremamente relevantes nos últimos anos para o estudo do favoritismo ingroup. Todavia, uma busca sistemática nas bases de dados Lilacs, Scielo, Google Scholar e Web of Science por palavras-chave relacionadas ao viés de grupo demonstrou uma carência por publicações nesse tema no Brasil. Dos trabalhos recuperados, apenas um se enquadrava na perspectiva evolucionista e nenhum abordava os mecanismos neuroendócrinos do viés de grupo. Dessa forma, o objetivo do presente estudo é discutir o viés de grupo através de uma perspectiva biológica, explicitando as hipóteses para a evolução desses comportamentos, os métodos empregados para o estudo dos mesmos e as bases neuroendócrinas e os substratos neurais que os medeiam.
\end{abstract}

Palavras-chave: Favoritismo ingroup, teoria dos jogos, neuroendocrinologia, perspectiva evolucionista.

\section{"In x Out": Reviewing the Group Bias through the Biological Perspective}

\begin{abstract}
The factors underlying to the ingroup favoritism and outgroup indifference/hostility are broadly studied by social psychology, where studies report that, for example, individuals trust more and associate positive words to members of the same group. The study of these factors can help in understand phenomena such as prejudice and ethonocentrism. However, a systematic search in the databases Web of Science, Google Scholar, Scielo, and Lilacs for the keywords related to group bias showed a studies shortage for this topic in Brazil. Besides, in the studies retrieved, just one fitted into evolutionary perspective and no study has approached the neuroendocrine mechanisms of the group bias. Therefore, the objective of this study was to discuss the group bias through the biological perspective, explaining the evolutionary hypothesis to the evolution of these behaviors, the methods applied to study this topic, and the neuroendocrine basis and neural substrates mediating them.
\end{abstract}

Keywords: Ingroup favoritism, game theory, neuroendocrinology, evolutionary perspective.

Endereço para correspondência: Universidade Federal do Rio Grande do Norte, Centro de Biociências, Caixa Postal 1511, Campus Universitário, Natal, RN, Brasil 59072970. Fone: (084) 215 3409. Fax: (084) 2119206. E-mail: victorshiramizu@gmail.com e emiliayamamoto@gmail.com 


\section{"In x Out": Revisando el Sesgo de Grupo a través de la Perspectiva Biológica}

\section{Resumen}

Los factores subyacentes al favorecimiento del endogrupo y a la indiferencia/hostilidad al exogrupo son ampliamente estudiados por la psicología social, donde estudios informan, por ejemplo, que los indivíduos confian más y asocian palabras positivas a los miembros del mismo grupo. El estúdio de esos factores puede ayudar en la comprensíon de fenómenos tales como el prejuicio y el etnocentrismo. Las perspectivas evolucionista y neuroendócrina han sido extremadamente relevantes en los últimos años para el estúdio del favoritismo ingroup. Sin embargo, una búsqueda sistemática en las bases de datos Lilacs, Scielo, Web of Science y Google Scholar por palabras claves relacionadas al sesgo de grupo demostró una carencia de publicaciones sobre ese tema en Brasil. De los trabajos recuperados sólo uno se ajustaba a la perspectiva evolucionista y ninguno abordaba los mecanismos neuroendocrino del sesgo de grupo. De esta manera, el objetivo del presente estudio es discutir el sesgo de grupo a través de una perspectiva biológica, explicando las hipótesis para la evolución de esos comportamientos, los métodos utilizados para el estúdio de ellos, las bases neuroendocrinas y los sustratos neurales que los median.

Palabras claves: Favoritismo ingroup, teoría de juegos, neuroendocrinología, perspectiva evolucionista.

Os comportamentos de favorecimento ao endogrupo, também conhecidos como favoritismo ingroup, e de indiferença/hostilidade ao exogrupo são amplamente estudados pela psicologia social para compreender fenômenos tais como preconceito e etnocentrismo (Brewer \& Kramer, 1985; Hewstone, Rubin \& Willis, 2002). Embora amplamente explorados, no Brasil, pesquisas investigando fatores que promovem o viés de grupo são escassos.

Realizamos um levantamento de trabalhos de conclusão de curso, dissertações de mestrado, teses de doutorado, artigos e capítulos de livros publicados no Brasil nos últimos cinco anos (do ano de 2011 até o mês de maio do ano de 2016) nas bases de dados Google Scholar, Lilacs, Scielo e Web of Science pelas seguintes palavras-chave: "Endogrupo/exogrupo" (correspondente em inglês Ingroup/outgroup), "Favoritismo ingroup", "Favorecimento do próprio grupo" e "Grupos mínimos". Ao final, 27 trabalhos ${ }^{2}$ foram incluídos, dos quais 12 foram artigos publicados em periódicos e somente um desses adotou a perspectiva evolucionista para a compreensão

2 As referências marcadas com um asterisco indicam os trabalhos recuperados na busca sistemática. do favoritismo ingroup. Além disso, nenhum desses trabalhos recuperados abordou o tópico de viés de grupo a partir de uma perspectiva neuroendócrina que ajudassem na compreensão de mecanismos causais. Tal perspectiva vem sendo extremamente relevante nos últimos anos (para uma revisão ver De Dreu, 2012) e uma revisão que estivesse disponível sobre esses achados poderia ser de grande relevância para a literatura aqui no país.

Dessa forma, nesse trabalho pretendemos revisar o viés de grupo a partir de uma perspectiva evolucionista, levando em consideração as principais teorias que buscam compreender sua evolução, os métodos adotados e os mecanismos neuroendócrinos que são modulados durante condições de conflito intergrupo.

\section{"Nós Versus Eles"}

Estudos clássicos no campo da psicologia social demonstraram o fato de que indivíduos são capazes de discriminar aqueles que pertencem ao próprio grupo e aqueles que não pertencem (Allport, 1954; Tajfel, Billig, Bundy, \& Flament, 1971). Esse processo de discriminação promove um fenômeno denominado de favori- 
tismo ingroup, o qual pode ser definido como o desejo de beneficiar alguém do mesmo grupo (Balliet, Wu, \& De Dreu, 2014). Estudos prévios exploraram as repercussões do viés de grupo, demonstrando que indivíduos: confiam mais em membros do ingroup quando expostos a uma situação de ameaça (Voci, 2006); associam palavras com conteúdo positivo ao próprio grupo (Perdue, Dovidio, Gurtman, \& Tyler, 1990); aumentam a quantidade de doações para o ingroup em situações de competição (Rubin, Badea, \& Jetten, 2014).

Uma vez que esse viés de grupo promove o favoritismo (altruísmo paroquial) a indivíduos do mesmo grupo, ele também pode resultar em sentimentos de evitação, menosprezo e hostilidade a indivíduos de outros grupos (Hewstone et al., 2002). Todavia, Brewer (1999) argumenta que o favoritismo do próprio grupo e o menosprezo/indiferença a outros grupos são independentes, sendo tal associação exacerbada em contextos de competição ou escassez de recursos. Além disso, acúmulo de evidências demonstra que a formação de um viés de grupo é causada muito mais por fatores que promovem o próprio grupo do que por fatores que menosprezem ou ameacem o outro grupo (para uma revisão, ver Balliet et al., 2014).

Interessantemente, tanto em condições naturalísticas quanto em configurações de laboratório, estudos demonstram que o favoritismo ingroup é flexível e dinâmico. Por exemplo, Tajfel et al. (1971) observaram que indivíduos escolhiam uma matriz de recompensas que maximizasse o lucro para o ingroup quando comparado com o outgroup. Vale salientar que esses indivíduos não tiveram nenhum contato face a face e nem conheciam os membros do mesmo grupo nem do outro grupo, somente foram informados que pertenciam ao mesmo grupo por um critério arbitrário, como um pretenso desempenho em um teste ou a preferência pelas obras de um pintor. Esse experimento ficou conhecido como paradigma dos grupos mínimos, demonstrando como uma característica pode ser flexível na formação de grupos. Rand et al. (2009) investigaram o quanto o favoritismo ingroup é dinâmico aproveitando-se das eleições presidenciais nos Estados Unidos de 2008. Utilizando um jogo econômico, os autores observaram que eleitores durante as eleições primárias entre candidatos Democratas Barack Obama e Hillary Clinton apresentaram um forte favoritismo ingroup. Todavia, esse efeito sumiu após as primárias e os grupos fundiram-se durante as eleições gerais.

A habilidade de avaliar e categorizar outra pessoa como "amiga" ou "inimiga" é fundamental para a sobrevivência em um ambiente social complexo (Hamlin, Wynn, \& Bloom, 2007). Evidências recentes demonstram que tal habilidade desenvolve-se precocemente em humanos. Em uma configuração de teatro infantil, Hamlin, Wynn e Bloom (2010) observaram que bebês, com três meses de idade, passaram a maior parte do tempo olhando para o boneco que ajudou o outro quando comparado com o que dificultou. Buttelmann e Böhm (2014) investigaram a ontogenia do viés de grupo em crianças de seis e oito anos, utilizando um jogo no qual as mesmas teriam a oportunidade de colocar figuras de natureza positiva ou negativa numa caixa do próprio grupo, do outro grupo ou em uma caixa neutra. Tanto crianças com seis quanto com oito anos de idade apresentaram favoritismo ingroup na alocação de figuras positivas. Interessantemente, crianças com seis anos de idade alocaram figuras negativas na caixa do outro grupo significativamente menos quando comparadas com crianças de oito anos de idade, sugerindo que o menosprezo pelo outgroup é mais acentuado após os seis anos de idade.

O desenvolvimento do viés de grupo é, no entanto, mais complexo do que pode parecer à primeira vista, mostrando uma elaborada interação entre predisposições biológicas e modulação cultural. Kinzler, Shutts e Correll (2010) revisam estudos que sugerem que sexo, idade e raça são categorias rapidamente identificadas por crianças muito jovens. No entanto, a categorização do sexo aparece mais cedo (3-4 anos) do que a de raça (4-5 anos) sugerindo, por um lado, que a categorização racial requer maior cognição e/ou aprendizado, e por outro, que a categorização do sexo tem um significado biológico mais importante do que a raça. Por exemplo, reconhecer cuidadores e mais tarde parceiros potenciais. 
Ainda enfatizando a maior relevância da categorização do sexo, Kurzban, Tooby e Cosmides (2001), mostraram que, através da manipulação da coalizão de grupo, era possível "apagar" a categorização de raça, mas não a de sexo.

\section{A Evolução do Favoritismo Ingroup}

Modelos utilizando simulação matemática buscam compreender a evolução do favoritismo ingroup. Hammond e Axelrod (2006) verificaram, em uma condição de etnocentrismo, que o favoritismo a membros do próprio grupo é um mecanismo bastante poderoso para promover cooperação na ausência de interações prolongadas e que o mesmo se torna mais evidente em ambientes hostis. Ainda, os autores defendem que esse mecanismo pode ser evolutivamente derivado de sistemas de reconhecimento de indivíduos aparentados.

Em outro estudo de simulação matemática, Choi e Bowles (2007) verificaram que, isoladamente, altruísmo ou hostilidade outgroup não teriam sido viáveis pelo elevado custo para o indivíduo. Todavia, grupos que se engajavam em mais ações altruísticas teriam mais vantagens quando competindo com grupos que não se engajavam em tais ações. Dessa forma, a força motriz para a evolução do favoritismo ingroup e do menosprezo/indiferença pelo outgroup teria sido o conflito entre grupos.

Por último, o favoritismo por membros do próprio grupo poderia ter evoluído em virtude do aumento da certeza que o ato altruístico seria retribuído no futuro. Assim, a probabilidade de interações futuras com membros do ingroup é maior quando comparada com membros do outgroup, sendo heuristicamente vantajoso cooperar preferencialmente com indivíduos do mesmo grupo (Kiyonari, Tanida, \& Yamagishi, 2000).

Kurzban et al. (2001) argumentam que ao longo da história evolutiva de humanos, os mesmos se depararam com cenários de competição entre grupos por recursos escassos, fato que demandou a categorização do ambiente social em "Nós versus eles". Esse mecanismo certamente apresentou vantagens adaptativas no ambiente de adaptação evolutiva por promover o favoritismo a membros do mesmo grupo e a hostilidade a membros de outros grupos. Portanto, o mecanismo de "Nós versus eles" promove o aumento da coesão do grupo.

A teoria da fusão de identidade busca compreender o motivo pelo qual indivíduos desenvolvem um sentimento visceral de unidade com o grupo e os membros do mesmo, tornando sua identidade pessoal e social funcionalmente equivalente (Swann, Gómez, Seyle, Morale, \& Huici, 2009). Além disso, os outros membros do grupo motivam comportamentos pró-sociais custosos e são vistos por aqueles extremamente fundidos como uma "família" (Swann, Jetten, Gómez, Whitehouse, \& Bastian, 2012). Interessantemente, quando a ativação fisiológica está alta, indivíduos extremamente fundidos são mais propensos a comportamentos pró-sociais extremos (Gómez et al., 2011). Mas qual seria a lógica evolutiva para indivíduos sacrificarem-se por um grupo de indivíduos não aparentados? Swann e Buhrmester (2015) argumentam que quando pessoas altamente fundidas percebem que membros do grupo compartilham um amplo conjunto de características, elas projetam mais frequentemente vínculos familiares a grupos maiores. Tal processo poderia fazer com que esses indivíduos fossem mais propensos a se sacrificarem por grupos heterogêneos.

\section{Compreendendo o Viés de Grupo Através da Teoria dos Jogos}

A teoria dos jogos passou a ser amplamente empregada para a compreensão do conflito e da cooperação em diferentes grupos e contextos, utilizando modelos matemáticos em que dois ou mais indivíduos tomam decisões, influenciando-se mutuamente e o lucro de cada um depende tanto da própria estratégia quanto da estratégia do outro (Alencar \& Yamamoto, 2008; Myerson, 1991). Do ponto de vista evolutivo, a seleção natural tende a manter na população genes dos "bons jogadores", ou seja, indivíduos que maximizam os ganhos e/ou minimizam as perdas, convertendo tais ganhos em aptidão (Rand \& Nowak, 2013; Smith, 1974). 
O cerne dos jogos econômicos é a simplicidade empregada, em que um indivíduo frequentemente apresenta uma estratégia dominante interesseira, a qual é fácil de compreender. Contudo, se o mesmo não adota tal estratégia interesseira, certamente pode-se inferir que ele teve algum motivo para não fazer, tal como crenças sobre confiança, reciprocidade ou expectativas de aplicação de regras sociais (Everett, Faber, \& Crockett, 2015; Fehr, Naef, \& Schmidt, 2006). Apresentaremos os principais jogos econômicos utilizados para a compreensão do viés de grupo e possíveis variáveis modulando tal comportamento a seguir.

\section{Jogo do Ditador}

Dois jogadores participam, sendo um deles (ditador) responsável por alocar uma quantidade de recurso (variando de toda a quantidade a nada) ao outro que deve aceitar a oferta. O recurso em questão pode variar de unidades monetárias reais a hipotéticas. Por exemplo, o ditador recebe 10 unidades monetárias e aloca destas 2 unidades, cabendo ao outro jogador apenas aceitar a divisão. Assim, o resultado do jogo do ditador pode ser interpretado como fonte primária de preferência social (Everett et al., 2015). Fowler e Kam (2007) utilizaram o jogo do ditador para verificar a quantidade de doações que republicanos e democratas fariam. Os autores observaram que democratas doaram significativamente mais ao seu grupo social. $\mathrm{O}$ mesmo foi verdade para republicanos.

\section{Jogo do Ultimato}

Semelhante ao jogo anterior, um jogador é responsável em alocar uma quantidade de recurso ao outro jogador e cabe a este aceitar ou não a oferta. Caso o mesmo rejeite, nenhuma dos dois recebe nada. Kubota, Li, Bar-David, Banaji e Phelps (2013) investigaram se o pertencimento a um grupo étnico contribuía na frequência de rejeição de uma divisão injusta no jogo do ultimato. Os mesmos observaram que os participantes, independente do grupo étnico, aceitaram mais propostas injustas de pessoas brancas do que de negros.

\section{Dilema do Prisioneiro}

Dois jogadores têm a opção de cooperar ou desertar. Caso ambos cooperem, eles conseguem um resultado positivo. Se um dos jogadores coopera e o outro deserta, o primeiro fica com o lucro mais alto e o outro, com o mais baixo. A opção mais vantajosa para uma única interação seria a de não cooperar. Todavia, para interações repetidas, os indivíduos ajustam essas decisões em função do comportamento do outro. Utilizando o dilema do prisioneiro reiterado, Yamagishi e Mifune (2009) verificaram o favoritismo pelo ingroup em estudantes universitários. Indivíduos cooperaram significativamente mais com indivíduos que pertenciam ao mesmo grupo do que com indivíduos do outro grupo, sendo tal efeito muito mais forte para homens do que para mulheres, demonstrando que esse mecanismo em homens favorece a solidariedade dentro do grupo.

\section{Jogo da Confiança}

Dois jogadores interagem, sendo o jogador A responsável em transferir alguma, toda ou nenhuma quantidade de recursos ao jogador B. Caso ocorra a transferência, o valor é multiplicado por três. O jogador B agora é responsável por retornar ou não a quantidade que ele julga ser adequada. Smith (2011) utilizou o paradigma dos grupos mínimos e observou que indivíduos confiaram mais naqueles pertencentes ao ingroup quando comparados com o outgroup durante o jogo da confiança.

\section{Jogo dos Bens Públicos}

Jogo composto por múltiplos jogadores, onde os mesmos devem decidir o quanto de recursos mantem para si mesmo ou o quanto de recursos é adicionado a um bem comum. A quantidade final existente no bem comum é multiplicada por um determinado fator e então dividida igualmente entre os jogadores. Parks, Sanna e Berel (2001) utilizaram o jogo dos bens públicos sendo manipulada a informação aos participantes de que os outros poderiam pertencer ao mesmo grupo ou a outro grupo. As taxas de doação para o bem público foram significati- 
vamente maiores quando os participantes eram informados que os outros pertenciam ao mesmo grupo, fato que demonstra uma clara preferência na doação para um recurso comum quando o mesmo é compartilhado pelo mesmo grupo.

\section{Mecanismos Neuroendócrinos Subjacentes ao Viés de Grupo}

No campo da neurociência social, o hormônio ocitocina ganhou o cenário como um dos mais importantes moduladores químicos do comportamento social (MacDonald \& MacDonald, 2010). Tal hormônio é produzido pelo núcleo paraventricular e supra-óptico do hipotálamo, sendo liberado através da hipófise posterior, caindo na grande circulação (Carter, 1998). Seus efeitos fisiológicos bem conhecidos são a facilitação do parto e a ejeção do leite. Receptores para ocitocina estão amplamente distribuídos em diversas regiões do cérebro associadas com comportamento sexual, formação do par, comportamento materno e a capacidade de formar vínculos emocionais (para uma revisão, ver De Dreu \& Kret, 2016).

Kosfeld, Heinrichs, Zak, Fischbacher e Fehr (2005) hipotetizaram que esse hormônio poderia aumentar a confiança em humanos durante uma situação de dilema social, uma vez que evidências em modelos animais demonstravam que a ocitocina promovia apego social e afiliação. Os autores administraram ocitocina intranasal ou placebo em homens, os quais participaram do jogo da confiança. Aqueles jogadores que receberam ocitocina transferiram muito mais unidades monetárias ao outro jogador. Isso demonstra que ocitocina é capaz de modular a confiança nas relações interpessoais.

No que diz respeito ao viés de grupo, De Dreu, Greer, Van Kleef, Shalvi e Handgraaf (2011) investigaram se a ocitocina modularia o etnocentrismo. Administrando ocitocina intranasal ou placebo, participantes de nacionalidade holandesa participaram de um teste de associação implícita. Aqueles que receberam ocitocina atribuíram palavras positivas muito mais rapidamente a nomes que remetiam a sua nacionalidade. Ainda, participantes foram submetidos a um dilema moral com a opção de salvar um grupo em detrimento da vida de um indivíduo. Comparado com o grupo placebo, o grupo ocitocina reduziu o sacrifício do ingroup e esse resultado não foi devido à hostilidade outgroup, uma vez que o tratamento com ocitocina não aumentou a prontidão para sacrificar indivíduos do outro grupo. Esse experimento foi de grande importância por demonstrar que a ocitocina aumenta o favoritismo ao próprio grupo.

Evidências também demonstram que a ocitocina modula a seleção de aliados para um possível conflito intergrupo. De Dreu, Greer, Handgraaf, Shalvi e Van Kleef (2012), novamente em um estudo com administração de ocitocina ou placebo em homens, observaram que indivíduos do grupo ocitocina reportaram que as fotos com faces com elevada ameaça seriam mais úteis como possíveis aliados.

Todavia, os estudos empregando ocitocina intranasal e suas repercussões no favoritismo ingroup são bastante questionáveis. $\mathrm{O}$ estudo metanalítico de Nave, Camerer e McCullough (2015) não encontrou evidência convergente para os efeitos moduladores da ocitocina na confiança em humanos, levando questões de se ocitocina intranasal pode alcançar o sistema nervoso central. Vale salientar que estudos prévios demonstram que as concentrações endógenas de ocitocina são moduladas por jogos econômicos, sem a necessidade de administração de ocitocina (Morhenn, Park, Piper, \& Zak, 2008; Zak, Kurzban, \& Matzner, 2005).

Além da ocitocina, outro hormônio explorado para melhor compreender o viés de grupo é a testosterona. Esse hormônio esteroide é produzido em homens principalmente nos testículos e, em mulheres, nas glândulas adrenais (Nelson $\&$ Trainor, 2007). Uma ampla literatura relaciona a testosterona com comportamento agressivo, dominância e atenção a ameaças (Archer, 2006).

Evidências demonstram que as concentrações de testosterona são moduladas durante jogos econômicos. O estudo de Burnham (2007) demonstrou que homens com maiores concentrações endógenas de testosterona recusaram significativamente mais ofertas injustas durante o jogo do ultimato, quando comparados com 
aqueles com menores concentrações. Interessantemente, mulheres que receberam testosterona sublingual fizeram ofertas mais justas durante o jogo do ultimato quando comparadas com o grupo controle (Eisenegger, Naef, Snozzi, Heinrichs, \& Fehr, 2010).

Van Honk, Montoya, Bos, van Vugt e Terburg (2012), utilizando o jogo dos bens públicos, verificaram que testosterona exógena aumentou a porcentagem de doações ao bem público. Esse efeito da testosterona na cooperação foi mais evidente em participantes com baixa exposição de testosterona no período pré-natal (verificada através da razão do segundo e quarto dígito da mão).

Recentemente, Reimers e Diekhof (2015) investigaram o papel da testosterona no favoritismo ingroup em fãs de futebol. Durante o dilema do prisioneiro, indivíduos com elevadas concentrações de testosterona aumentaram a cooperação com o ingroup quando o contexto era de competição entre grupos. Esses resultados demonstram que as concentrações de testosterona são moduladas tanto no contexto de jogos econômicos quanto durante o conflito entre grupos.

Esses estudos demonstram que mecanismos neuroendócrinos são recrutados frente a situações de trocas sociais, bem como situações de favorecimento do próprio grupo, podendo exacerbar ou atenuar respostas comportamentais.

\section{O Cérebro em Viés}

Estudos com ressonância magnética funcional (fMRI) investigam as bases neurais do favorecimento do viés de grupo. Volz, Kessler e von Cramon (2009), utilizando o paradigma de grupos mínimos, solicitaram que os participantes alocassem uma quantidade de dinheiro para o próprio grupo ou para o outro grupo. Os autores observaram que indivíduos apresentando um forte viés de grupo tinham uma maior ativação em duas áreas corticais: o córtex pré-frontal medial e o córtex cingulado póstero-anterior. Os mesmos hipotetizaram que a primeira área é responsável pela "identidade social", ou seja, indivíduos pensam em atributos pessoais positivos que os fazem identificar-se com o grupo social, levando-os a expressar um maior favoritismo pelo ingroup. Já a segunda área, é responsável por integrar informações entre o sistema límbico e o córtex pré-frontal e por participar da regulação afetiva, proporcionando o famoso "colorido emocional", devido a esses processos de integração, resultando na evocação de emoções positivas ou negativas (Stevens, Hurley, \& Taber, 2011; Volz et al., 2009). Assim, tal circuitaria neural parece proporcionar ao indivíduo uma maior facilidade em realizar associações positivas ao ingroup.

Se indivíduos distinguem membros do próprio grupo quando comparados com os de outro grupo, processos perceptuais podem funcionar diferentemente. Molenberghs, Halász, Mattingley, Vanman e Cummington (2013) utilizaram uma condição em que dois grupos competiam para ver quem executava uma atividade num menor tempo. Os participantes assistiram aos vídeos dessa atividade, os quais foram manipulados para que o tempo dos grupos fosse igual, enquanto a atividade cerebral foi registrada utilizando ressonância magnética funcional. Participantes reportaram que membros do ingroup executaram a tarefa mais rapidamente quando comparados com membros do outgroup e, interessantemente, apresentaram uma maior atividade do lobo parietal inferior durante esse processo, sendo tal área crucial para o acoplamento de informações entre representações visuais e o córtex motor, sugerindo que o viés de grupo promove uma percepção visual diferenciada aos membros do mesmo grupo.

Estudos reportam que indivíduos são mais empáticos com membros do próprio grupo (para uma revisão, ver De Dreu \& Kret, 2016). Para investigar os mecanismos neurais subjacentes à empatia expressa durante o viés de grupo, Hein, Silani, Preuschoff, Batson e Singer (2010) conduziram um experimento no qual fãs de futebol observavam outros fãs do mesmo time ou do time rival levando pequenos choques durante um intervalo de tempo. Participantes reportaram uma maior propensão a dividir o tempo de choque com torcedores do mesmo time de futebol e que tal propensão estava associada com uma 
maior ativação da ínsula anterior. Essa região está relacionada à empatia e compreensão de estados do outro. Surpreendentemente, quando observando torcedores do time rival sentindo dor, participantes reportaram uma menor propensão a ajudar e essa medida correlacionou-se com uma maior atividade do núcleo accumbens.

Ainda, pesquisas têm investigado quais áreas neurais poderiam estar envolvidas em um processamento diferenciado no que diz respeito a faces de indivíduos do ingroup e do outgroup. Por exemplo, Cunningham et al. (2004) apresentaram a participantes caucasianos vídeos de curta (processamento automático) e longa duração (processamento controlado) com faces de brancos e negros. Quando expostos a vídeos curtos com faces de negros, os participantes tiveram uma ativação significativamente maior da amígdala, área do sistema límbico associada com resposta emocional. Já quando expostos a vídeos de longa duração, tal diferença foi significativamente reduzida, ao passo que uma maior atividade do córtex frontal foi encontrada, área relacionada com controle e inibição.

Lieberman, Hariri, Jarcho, Eisenberger e Bookheimer (2005) observaram o mesmo padrão de ativação na amígdala quando apresentando fotos de afro-americanos e americanos caucasianos para as mesmas etnias. Todavia, esse efeito foi reduzido quando a etnia foi processada de forma verbal, onde o córtex pré-frontal ventromedial foi à área mais recrutada, área que possui projeções inibitórias para a amígdala. Esses achados sugerem que tal categorização parece ser culturalmente aprendida e que sua expressão pode ser, também culturalmente, inibida.

Os estudos acima citados fornecem evidências de circuitos neurais específicos que são ativados: para o reconhecimento de faces de indivíduos do próprio grupo; para uma percepção diferenciada às atividades de membros do próprio grupo; bem como regiões associadas à empatia.

\section{Considerações Finais}

O favoritismo por grupos de pertinência e a formação dessas preferências é um tema há muito estudado pela Psicologia, desde os trabalhos pioneiros de Allport (1954) e Tajfel et al. (1971). No entanto, este assunto tem sido pouco estudado no Brasil, seja pela Psicologia Social, origem dos trabalhos pioneiros, seja pela Psicologia Evolucionista (Brewer, 1999; Brewer \& Kramer, 1985), ou a Psicologia Fisiológica e as Neurociências (De Dreu \& Kret, 2016; Hein et al., 2010) que mais recentemente tem abordado esta questão. A falta de estudos brasileiros deste tema é surpreendente, uma vez que nossa sociedade apresenta sérios problemas de desigualdade social, discriminação e violência que frequentemente estão relacionados a atitudes preconceituosas e de exclusão social. Acreditamos que estas questões poderiam ser melhor compreendidas utilizando este referencial teórico, pois a percepção de pertinência a um grupo é um forte mecanismo de cooperação, mas também de discriminação (Boyer, 2001; Cosmides, Tooby \& Kurzban, 2003).

No estudo desta questão a perspectiva evolucionista esclarece aspectos pouco investigados que dizem respeito às possíveis razões para o aparecimento e manutenção do favoritismo dirigido ao endogrupo na espécie humana, suas vantagens adaptativas. A socialidade, embora característica não apenas da espécie humana, mas da maior parte dos primatas, é, em nossa espécie, mediada pela cognição, o que favorece a coordenação de comportamento e estratégias. Ao longo da evolução, não apenas a coordenação de comportamento favoreceu a cooperação, mas principalmente a seleção de parceiros preferenciais - membros do endogrupo.

Nesse sentido, indivíduos que favoreciam seu próprio grupo foram mais bem sucedidos, o que deu ocasião ao aparecimento e fortalecimento de mecanismos psicológicos que predispõem esse favorecimento pelos seres humanos, e que ocorrem desde muito cedo no desenvolvimento, como discutido anteriormente.

Porém, predisposições não predizem o comportamento em qualquer situação e contexto comportamentos, do ponto de vista evolutivo, são o produto da história de nossa espécie, mas também são moduladas pelo ambiente, presente e de desenvolvimento. Basta lembrar que embo- 
ra alguns grupos tenham significância biológica obrigatória, como os grupos familiares, a maioria dos grupos dos quais fazemos parte são construídos socialmente, como os grupos políticos e religiosos. Cabe a nós reconhecer que essas predisposições estão presentes e procurar as condições nas quais elas podem promover a aceitação e o respeito dos que são diferentes de nós e as oportunidades de cooperação. Acreditamos que a perspectiva evolucionista pode contribuir nesse sentido.

\section{Referências}

Alencar, A. I., \& Yamamoto, M. E. (2008). A teoria dos jogos como metodologia de investigação científica para a cooperação na perspectiva da psicologia evolucionista. Psico, 39(4), 3.

Allport, G. W. (1954). The nature of prejudice. New York: Addison Wesley.

*Almeida, S. S. M. (2012). Análise do autoconceito e autocontrole de crianças negras a partir da identidade social (Dissertação de mestrado, Universidade Federal de Sergipe, Aracajú, SE, Brasil).

*Amorim, C. R. (2014). Religiões afro-brasileiras e identidades: Interlocuções entre Antropologia e Psicologia Social. (Syn) thesis, 7(2), 159-168.

*Aquino, T. A. A., Gouveia, V., da Silva, J. P., \& da Silva, D. M. (2013). Correlatos valorativos do preconceito religioso: Um estudo em uma escola pública estadual. Interação em Psicologia, 17(3), 259-269.

Archer, J. (2006). Testosterone and human aggression: An evaluation of the challenge hypothesis. Neuroscience \& Biobehavioral Reviews, 30(3), 319-345.

Balliet, D., Wu, J., \& De Dreu, C. K. (2014). Ingroup favoritism in cooperation: A meta-analysis. Psychological Bulletin, 140(6), 1556.

*Batista, J. R. M. (2014). Os estereótipos e o efeito do contato virtual no preconceito contra negros e nordestinos (Dissertação de mestrado, Universidade Federal da Paraíba, João Pessoa, PB: Brasil).

*Bonomo, M., Trindade, Z. A., Souza, L. D., \& Coutinho, S. M. D. S. (2008). Representações sociais e identidade em grupos de mulheres ciganas e rurais. Psicologia, 22(1), 153-181.
Boyer, P. (2001). Religion explained: The evolutionary origins of religious thought. New York: Basic Books.

*Brasil, J. A. (2012). Latino-americanos e europeus no Brasil: Analisando processos migratórios e identitários no estado do Espírito Santo (Tese de doutorado, Universidade Federal do Espírito Santo, Vitória, ES, Brasil).

Brewer, M. B. (1999). The psychology of prejudice: Ingroup love and outgroup hate?. Journal of Social Issues, 55(3), 429-444.

Brewer, M. B., \& Kramer, R. M. (1985). The psychology of intergroup attitudes and behavior. Annual Review of Psychology, 36(1), 219-243.

Burnham, T. C. (2007). High-testosterone men reject low ultimatum game offers. Proceedings of the Royal Society of London B: Biological Sciences, 274(1623), 2327-2330.

Buttelmann, D., \& Böhm, R. (2014). The ontogeny of the motivation that underlies in-group bias. Psychological Science, 25(4), 921-927.

Carter, C. S. (1998). Neuroendocrine perspectives on social attachment and love. Psychoneuroendocrinology, 23(8), 779-818.

Choi, J. K., \& Bowles, S. (2007). The coevolution of parochial altruism and war. Science, 318, 636640.

*Ciscon-Evangelista, M. R., \& Menandro, P. R. M. (2011). Trânsito religioso e construções identitárias: Mobilidade social de evangélicos neopentecostais. Psico USF, 16(2), 193-202.

Cosmides, L., Tooby, J., \& Kurzban, R. (2003). Perceptions of race. Trends in Cognitive Sciences, 7(4), 173-179.

Cunningham, W. A., Johnson, M. K., Raye, C. L., Gatenby, J. C., Gore, J. C., \& Banaji, M. R. (2004). Separable neural components in the processing of black and white faces. Psychological Science, 15(12), 806-813.

De Dreu, C. K. (2012). Oxytocin modulates cooperation within and competition between groups: An integrative review and research agenda. Hormones and behavior, 61(3), 419-428.

De Dreu, C. K., Greer, L. L., Van Kleef, G. A., Shalvi, S., \& Handgraaf, M. J. (2011). Oxytocin promotes human ethnocentrism. Proceedings of the National Academy of Sciences, 108(4), $1262-1266$ 
De Dreu, C. K., Greer, L. L., Handgraaf, M. J., Shalvi, S., \& Van Kleef, G. A. (2012). Oxytocin modulates selection of allies in intergroup conflict. Proceedings of the Royal Society of London B: Biological Sciences, 279, 1150-1154.

De Dreu, C. K., \& Kret, M. E. (2016). Oxytocin conditions intergroup relations through upregulated in-group empathy, cooperation, conformity, and defense. Biological Psychiatry, 79(3), 165-173.

* Di Castro, V. C. (2014). Sobreviventes da fusão e categorias sociais: Um estudo comparativo acerca do fenômeno das fusões (Dissertação de mestrado, Pontifícia Universidade Católica de Goiás, Goiânia, GO, Brasil).

Eisenegger, C., Naef, M., Snozzi, R., Heinrichs, M., $\&$ Fehr, E. (2010). Prejudice and truth about the effect of testosterone on human bargaining behaviour. Nature, 463(7279), 356-359.

Everett, J. A., Faber, N. S., \& Crockett, M. (2015). Preferences and beliefs in ingroup favoritism. Frontiers in Behavioral Neuroscience, 9(15), 1-21.

*Fehlberg, J. (2011). Trabalho, igreja e boteco: Identidades em transformação entre descendentes de Pomeranos do interior do Espírito Santo (Tese de doutorado, Universidade Federal do Espírito Santo, Vitória, ES, Brasil).

Fehr, E., Naef, M., \& Schmidt, K. M. (2006). Inequality aversion, efficiency, and maximin preferences in simple distribution experiments: Comment. The American Economic Review, 96(5), 1912-1917.

*Ferreira, F. M. (2012). Estereótipos e discurso: $A$ (re) construção da imagem de minorias em revistas de nicho (Tese de doutorado, Universidade de São Paulo, SP, Brasil).

*Fioravanti-Bastos, A. C. M., Seidl-de-Moura, M. L., \& Landeira-Fernandez, J. (2014). O desenvolvimento do efeito da outra raça (EOR) em crianças: Dos modelos de codificação de faces à emergência do EOR. Psicologia: Reflexão $e$ Crítica, 27(1), 134-144.

Fowler, J. H., \& Kam, C. D. (2007). Beyond the self: Social identity, altruism, and political participation. Journal of Politics, 69(3), 813-827.

*Gomes, C. S. (2015). Imagens meméticas e a comunic (ação) na web: Um experimento analítico, reflexões e aplicabilidades acerca do discurso multimodal do Facebook. Texto Livre: Linguagem e Tecnologia, 8(2), 54-68.
Gómez, A., Brooks, M. L., Buhrmester, M. D., Vázquez, A., Jetten, J., \& Swann, W. B., Jr. (2011). On the nature of identity fusion: Insights into the construct and a new measure. Journal of Personality and Social Psychology, 100(5), 918.

Hamlin, J. K., Wynn, K., \& Bloom, P. (2007). Social evaluation by preverbal infants. Nature, 450(7169), 557-559.

Hamlin, J. K., Wynn, K., \& Bloom, P. (2010). Threemonth-olds show a negativity bias in their social evaluations. Developmental Science, 13(6), 923929.

Hammond, R. A., \& Axelrod, R. (2006). The evolution of ethnocentrism. Journal of Conflict Resolution, 50(6), 926-936.

Hein, G., Silani, G., Preuschoff, K., Batson, C. D., \& Singer, T. (2010). Neural responses to ingroup and outgroup members' suffering predict individual differences in costly helping. $\mathrm{Neu}$ ron, 68(1), 149-160.

*Herrera, F. J. R. (2015). Desenvolvimento de valores sociais na perspectiva da psicologia semiótica-cultural: Um estudo com meninos brasileiros e colombianos em contexto lúdico sugestivo de violência (Tese de doutorado, Universidade de Brasília, DF, Brasil).

Hewstone, M., Rubin, M., \& Willis, H. (2002). Intergroup bias. Annual Review of Psychology, 53(1), 575-604.

Kinzler, K. D., Shutts, K., \& Correll, J. (2010). Priorities in social categories. European Journal of Social Psychology, 40(4), 581-592.

*Kirschbaum, C. (2012). Rappers em São Paulo: Conexões, disconexões e transgressões. Redes: Revista Hispana para el Análisis de Redes Sociales, 22, 5-22.

Kiyonari, T., Tanida, S., \& Yamagishi, T. (2000). Social exchange and reciprocity: Confusion or a heuristic?. Evolution and Human Behavior, 21(6), 411-427.

Kosfeld, M., Heinrichs, M., Zak, P. J., Fischbacher, U., \& Fehr, E. (2005). Oxytocin increases trust in humans. Nature, 435(7042), 673-676.

Kubota, J. T., Li, J., Bar-David, E., Banaji, M. R., $\&$ Phelps, E. A. (2013). The price of racial bias intergroup negotiations in the Ultimatum Game. Psychological Science, 24(12), 2498-2504.

Kurzban, R., Tooby, J., \& Cosmides, L. (2001). Can race be erased? Coalitional computation and so- 
cial categorization. Proceedings of the National Academy of Sciences, 98(26), 15387-15392.

Lieberman, M. D., Hariri, A., Jarcho, J. M., Eisenberger, N. I., \& Bookheimer, S. Y. (2005). An fMRI investigation of race-related amygdala activity in African-American and Caucasian-American individuals. Nature Neuroscience, $8(6), 720-722$.

*Luquiari, G. A. (2013). Agressividade: A origem dos conflitos?: Compreendendo o comportamento agressivo [Monografia]. Águas Claras, SP: Universidade Estadual de São Paulo.

MacDonald, K., \& MacDonald, T. M. (2010). The peptide that binds: A systematic review of oxytocin and its prosocial effects in humans. Harvard Review of Psychiatry, 18(1), 1-21.

*Maffezzolli, E. C. F., \& Prado, P. H. M. (2013). Identificação com a marca: Proposição de um instrumento de medida. REAd. Revista Eletrônica de Administração (Porto Alegre), 19(3), 588-619.

*Martins, T. A. A. (2015). Prática Pedagógica Libertadora: Princípios para superação de estereótipos culturais no ensino-aprendizagem de língua inglesa (Dissertação de mestrado, Universidade Estadual do Sudoeste da Bahia, Vitória da Conquista, BA, Brasil).

*Matos, T. G. R., Maia, L. M., \& Maciel, R. H. (2012). Catadores de material reciclável e identidade social: Uma visão a partir da pertença grupal. Interação em Psicologia, 16(2), 239-247.

*Modesto, J. G. N. (2014). "Nem todas as vítimas importam": A influência das crenças no mundo justo na responsabilização de vítimas de diferentes grupos sociais (Dissertação de mestrado, Universidade de Brasília, DF, Brasil).

Molenberghs, P., Halász, V., Mattingley, J. B., Vanman, E. J., \& Cunnington, R. (2013). Seeing is believing: Neural mechanisms of action-perception are biased by team membership. Human Brain Mapping, 34(9), 2055-2068.

Morhenn, V. B., Park, J. W., Piper, E., \& Zak, P. J. (2008). Monetary sacrifice among strangers is mediated by endogenous oxytocin release after physical contact. Evolution and Human Behavior, 29(6), 375-383.

*Mourão, R. V. C. B. (2012). Fatores que influenciam a cooperação em humanos (Tese de doutorado, Universidade Federal do Rio Grande do Norte, Natal, RN, Brasil).
Myerson, R. B. (1991). Game Theory: Analysis of conflict. Cambridge, MA: Harvard University Press.

Nave, G., Camerer, C., \& McCullough, M. (2015). Does oxytocin increase trust in humans? A critical review of research. Perspectives on Psychological Science, 10(6), 772-789.

Nelson, R. J., \& Trainor, B. C. (2007). Neural mechanisms of aggression. Nature Reviews Neuroscience, 8(7), 536-546.

*Oliveira, E. B. D. (2015). A influência da relevância social no viés de grupo (Dissertação de mestrado, Universidade Federal do Rio Grande do Norte, Natal, RN, Brasil).

*Oliveira, L. A. (2011). "Mate um nordestino afogado" - Análise crítica de um artigo da revista Época. Linguagem em (Dis) curso, 11(2), 361376.

Parks, C. D., Sanna, L. J., \& Berel, S. R. (2001). Actions of similar others as inducements to cooperate in social dilemmas. Personality and Social Psychology Bulletin, 27(3), 345-354.

*Peixoto, L. S. A., \& Gondim, S. M. G (2014). Saliência da identidade profissional ou de equipe: Estudo qualitativo com equipes multidisciplinares. Revista Brasileira de Psicologia, 1, 3-12.

Perdue, C. W., Dovidio, J. F., Gurtman, M. B., \& Tyler, R. B. (1990). Us and them: Social categorization and the process of intergroup bias. Journal of Personality and Social Psychology, $59(3), 475$

Rand, D. G., \& Nowak, M. A. (2013). Human cooperation. Trends in cognitive sciences, 17(8), 413-425.

Rand, D. G., Pfeiffer, T., Dreber, A., Sheketoff, R. W., Wernerfelt, N. C., \& Benkler, Y. (2009). Dynamic remodeling of in-group bias during the 2008 presidential election. Proceedings of the National Academy of Sciences, 106(15), 61876191.

Reimers, L., \& Diekhof, E. K. (2015). Testosterone is associated with cooperation during intergroup competition by enhancing parochial altruism. Frontiers in Neuroscience, 9, 183.

Rubin, M., Badea, C., \& Jetten, J. (2014). Low status groups show in-group favoritism to compensate for their low status and compete for higher status. Group Processes \& Intergroup Relations, 17(5), 563-576. 
*Sacco, A. M. (2015). Orgulho e preconceito: $O$ desenvolvimento de atitudes raciais implícitas e explícitas em crianças de Porto Alegre e Salvador (Tese de doutorado, Universidade Federal do Rio Grande do Sul, Porto Alegre, RS, Brasil).

*Santos, L. B. D. (2013). Preconceito, discriminação, luso-tropicalismo, lusofonia e justiça organizacional em Portugal, do ponto de vista de imigrantes brasileiros. Revista Psicologia Organizações e Trabalho, 13(1), 61-74.

*Simai, S., \& Baeninger, R. (2011). Conflito intragrupal na imigração internacional no Brasil: Análise comparativa sobre a imigração boliviana $\mathrm{e}$ a imigração haitiana de estudantes. In E. J. P. Prado \& R. Coelho (Eds.), Migrações e trabalho (pp. 203-222). Brasília, DF: Ministério Público do Trabalho.

Smith, A. (2011). Identifying in-group and out-group effects in the trust game. The BE Journal of Economic Analysis \& Policy, 11(1).

Smith, J. M. (1974). The theory of games and the evolution of animal conflicts. Journal of Theoretical Biology, 47(1), 209-221.

*Souza, L. E. C. D. (2014). A influência da categorização pelo sotaque na discriminação (Tese de doutorado, Universidade Federal da Paraíba, João Pessoa, PB, Brasil).

Stevens, F. L., Hurley, R. A., \& Taber, K. H. (2011). Anterior cingulate cortex: Unique role in cognition and emotion. The Journal of neuropsychiatry and Clinical Neurosciences, 23(2), 120-125.

Swann, W. B., \& Buhrmester, M. D. (2015). Identity fusion. Current Directions in Psychological Science, 24(1), 52-57.

Swann, W. B., Jr., Gómez, A., Seyle, D. C., Morales, J., \& Huici, C. (2009). Identity fusion: The interplay of personal and social identities in extreme group behavior. Journal of Personality and Social Psychology, 96(5), 995.
Swann, W. B., Jr., Jetten, J., Gómez, A., Whitehouse, H., \& Bastian, B. (2012). When group membership gets personal: A theory of identity fusion. Psychological Review, 119(3), 441.

Tajfel, H., Billig, M. G., Bundy, R. P., \& Flament, C. (1971). Social categorization and intergroup behaviour. European Journal of Social Psychology, 1(2), 149-178.

Van Honk, J., Montoya, E. R., Bos, P. A., van Vugt, M., \& Terburg, D. (2012). New evidence on testosterone and cooperation. Nature, 485(7399), E4-E5.

Voci, A. (2006). The link between identification and in-group favouritism: Effects of threat to social identity and trust-related emotions. British Journal of Social Psychology, 45(2), 265-284.

Volz, K. G., Kessler, T., \& von Cramon, D. Y. (2009). In-group as part of the self: In-group favoritism is mediated by medial prefrontal cortex activation. Social Neuroscience, 4(3), 244-260.

Yamagishi, T., \& Mifune, N. (2009). Social exchange and solidarity: In-group love or out-group hate? Evolution and Human Behavior, 30(4), 229-237.

Zak, P. J., Kurzban, R., \& Matzner, W. T. (2005). Oxytocin is associated with human trustworthiness. Hormones and Behavior, 48(5), 522-527.

Recebido: 20/06/2016

$1^{a}$ revisão: 09/12/2016 Aceite final: 26/12/2016 


\section{Apêndice}

\section{Tabela 1}

Resultados Recuperados de Acordo com os Critérios de Inclusão Aplicados entre 2011 e Maio de 2016

\begin{tabular}{|c|c|c|c|c|}
\hline Autor(es) & Base & Ano & Tipo & Palavra-chave \\
\hline Herrera & Google scholar & 2015 & Tese & Endogrupo/exogrupo \\
\hline Luquiari & Google scholar & 2013 & Monografia & Endogrupo/exogrupo \\
\hline Ferreira & Google scholar & 2012 & Dissertação & Endogrupo/exogrupo \\
\hline Oliveira & Google scholar & 2011 & Artigo & Endogrupo/exogrupo \\
\hline Santos & Lilacs & 2013 & Artigo & Endogrupo/exogrupo \\
\hline Bonomo, Souza, Menandro, \& Trindade & Scielo & 2011 & Artigo & Endogrupo/exogrupo \\
\hline Ciscon-Evangelista, \& Menandro & Scielo & 2011 & Artigo & Endogrupo/exogrupo \\
\hline Oliveira & Google scholar & 2015 & Dissertação & Favoritismo ingroup \\
\hline Simai \& Baeninger & Google scholar & 2015 & Livro & Favoritismo ingroup \\
\hline Modesto & Google scholar & 2014 & Dissertação & Favoritismo ingroup \\
\hline Peixoto \& Gondim & Google scholar & 2014 & Artigo & Favoritismo ingroup \\
\hline Amorim & Google scholar & 2014 & Artigo & Favoritismo ingroup \\
\hline Souza & Google scholar & 2014 & Tese & Favoritismo ingroup \\
\hline $\begin{array}{l}\text { Fioravanti-Bastos, Seidl-de-Moura, } \\
\text { \& Landeira-Fernadez }\end{array}$ & Google scholar & 2014 & Artigo & Favoritismo ingroup \\
\hline Di Castro & Google scholar & 2014 & Dissertação & Favoritismo ingroup \\
\hline Brasil & Google scholar & 2012 & Dissertação & Favoritismo ingroup \\
\hline Mourão & Google scholar & 2012 & Tese & Favoritismo ingroup \\
\hline Matos, Maia, \& Maciel & Google scholar & 2012 & Artigo & Favoritismo ingroup \\
\hline Almeida & Google scholar & 2012 & Dissertação & Favoritismo ingroup \\
\hline Martins & Google scholar & 2015 & Dissertação & $\begin{array}{l}\text { Favorecimento } \\
\text { do próprio grupo }\end{array}$ \\
\hline Batista & Google scholar & 2014 & Tese & $\begin{array}{l}\text { Favorecimento } \\
\text { do próprio grupo }\end{array}$ \\
\hline Aquino, Gouveia, da Silva, \& da Silva & Google scholar & 2013 & Artigo & $\begin{array}{l}\text { Favorecimento } \\
\text { do próprio grupo }\end{array}$ \\
\hline Gomes & Google scholar & 2015 & Artigo & Grupos mínimos \\
\hline Sacco & Google scholar & 2015 & Tese & Grupos mínimos \\
\hline Maffezzolli \& Prado & Google scholar & 2013 & Artigo & Grupos mínimos \\
\hline Kirschbaum & Google scholar & 2012 & Artigo & Grupos mínimos \\
\hline Fehlberg & Google scholar & 2011 & Tese & Grupos mínimos \\
\hline
\end{tabular}

